\title{
Unilateral striatal lesions in the cat disrupt well-learned motor plans in a GO/NO-GO reaching task
}

Received: 19 December 1995 / Accepted: 29 July 1996

\begin{abstract}
We examined the changes in learned and spontaneous motor behavior after a unilateral excitotoxin lesion of the neostriatum. Cats were trained to perform a sensory-cued GO/NO-GO reaching task. Success rate, reaction time, movement speed and kinematic patterns were used to characterize motor system properties. In addition, motor properties before and after the lesion were compared by clinical neurological examinations and video tape observations of free-range behavior. We found that in normal animals motor performance in the task was fluent, highly automatic and skillful with consistent patterns from trial to trial and day to day. The striatal lesion resulted in a marked impairment in the animals' ability to perform the automatic response to the sensory cues in the motor task. In contrast, sensorimotor behavior in contexts apart from the task was altered minimally, with changes that were often difficult to detect. The animals recovered their ability to perform the task gradually, although they never reached prelesion performance levels in up to 24 weeks of evaluation. The animals had difficulty making reaching movements in GO trials and, in NO-GO trials failures to withhold movements were more frequent. Failures were due to a specific inability to execute previously well-learned movements in response to cues and not to an inability to recognize and interpret the cues. The lesion effects were restricted to the automatic motor response to the learned cues, as the animals could make reaching movements to the target without obvious impairment in response to novel stimuli. They also made similar spontaneous movements apart from the motor task that appeared to be unimpaired. The unique motor style and strategies that characterized the behavior of individual animals prior to the lesion were still evident after the lesion, even though they were superimposed on lower success rates and slower movement speeds. Our findings suggest that the basal ganglia facilitate the fluent and rapid execution
\end{abstract}

J.W. Aldridge ( J.F. Thompson · S. Gilman

Department of Neurology, University of Michigan,

1103 East Huron, Ann Arbor, MI 48104-1687, USA;

Tel: + 1 (313)-763-3706, Fax: +1 (313)-764-2189,

e-mail: wayne.aldridge@umich.edu of sequences of well-learned sensorimotor behavior, but the representations of motor plans are not stored in the basal ganglia.

Key words Movement $\cdot$ Basal ganglia $\cdot$ Striatum . Reaction time $\cdot$ Sensory cues

\section{Introduction}

It is well known that damage to the basal ganglia in humans and animals will produce impairments of motor control. However, the exact role that the basal ganglia play in controlling movements remains elusive. The striatum is a key input structure of the basal ganglia with up to five functional subsystems (Alexander et al. 1986). The striatum receives a sizable projection from motor areas of the cerebral cortex, but prefrontal, cingulate and temporal cortical projections (Nauta 1986) suggest an additional contribution to the integrative aspects of motor behavior. Movement-related information processed by the basal ganglia completes the loop through pallidal projections in the thalamus (Parent and Hazrati 1995) to supplementary motor cortex (Schell and Strick 1984), a cortical region thought to have a role in movement planning (Roland 1980), and other motor and non-motor brain regions (Hoover and Strick 1993; Inase and Tanji 1995). Neuronal activity in these basal ganglia pathways is correlated to sensorimotor behavior (reviewed in DeLong et al. 1984), including parameters such as the force and velocity of movements (Georgopoulos et al. 1983), muscle activity (Anderson and Horak 1985) and cues that initiate movements (Aldridge et al. 1980a), although no single motor parameter can account for neuronal activity (Mink and Thach 1991) and movement-related changes of neuronal activity often follow rather than precede the onset of movements (Aldridge et al. 1980a, b). Neuronal activity in these pathways may be influenced preferentially by global and abstract properties of movement preparation and execution (Alexander and Crutcher 1990; Brotchie et al. 1991; Jaeger et al. 1995). Together, 
the anatomically indirect nature of basal ganglia contacts with the motor system and the tenuous relationship of neuronal activity to the details of movement suggest a supplementary, modulatory or supporting role in motor behavior rather than direct control of movements.

A supporting or facilitatory role in motor behavior is also suggested by studies of damage in basal ganglia caused by diseases in humans or experimental lesions in animals. In spite of generally slow and impoverished movement, patients with Parkinson's disease can learn and perform new movements (Stelmach et al. 1986). They have particular difficulty in rapidly and efficiently executing a sequences of movements (Benecke et al. 1987). In cats with large bilateral ablations of the caudate nucleus, Villablanca and his colleagues (1976) observed no obvious neurological deficits except "compulsory approaching" to the most intense sensory stimulus in the environment. They suggested that the animals were unable to modulate sensory inputs for the purposes of movement control. Lesions of the striatum in rodents also disrupt motor performance and increase reaction time (Amalric and Koob 1987; Brown and Robbins 1991). Localized striatal lesions in rats specifically impair stereotyped sequences of movements, although the execution of the individual components is unimpaired (Berridge and Fentress 1987).

Previous studies of unilateral striatal lesions have conflicting findings. A thorough study employing ablative lesions of the caudate nucleus in cats produced no detectable change in behavior (Villablanca et al. 1976). Another study with restricted electrolytic lesions showed short term athetoid- and choreiform-like hyperkinesia (Liles and Davis 1969). Excitotoxic striatal lesions, which have the advantage of sparing fibers of passage (Divac et al. 1978), also had no detectable effect on freerange motor behavior in spite of nearly complete neuronal destruction in the striatum and clear-changes in neuronal activity in deafferented structures (Sachdev et al. 1991). If the basal ganglia are facilitating movements rather than delineating the details, it is possible that not all motor behavior would invoke basal ganglia participation, and this may explain why some studies show little effect. There is evidence for the susceptibility of particular types of motor behavior in Parkinson's and Huntington's disease. Well-learned, procedural motor tasks that are executed automatically seem to be especially vulnerable (Saint-Cyr et al. 1988; Knopman and Nissen 1991). Thus, highly practiced sequences of movement may be more likely to recruit the participation of the basal ganglia. In this study, we tested this idea by training cats to perform a sensorimotor task. The animals were given extensive practice to produce consistent and automatic motor performance. We explored the changes in motor behavior resulting from well-delineated and complete unilateral lesions of the striatum. Although there was little change in free-range behavior, a permanent and devastating deficit on the highly practiced motor task was apparent. Similar movements in contexts apart from the highly practiced motor task were not impaired.

\section{Methods}

Animals

Four domestic cats were used; two received striatal lesions after prelesion data were collected. The animals were trained to perform a motor task described below. For neuronal recording, a stainless steel chamber was secured (Aldridge et al. 1988) to the skull with bone screws and dental acrylic under general anesthesia in a location to provide access to the basal ganglia. The animals were allowed 2 weeks to recover from surgery before testing began. Animal use procedures were developed in consultation with the personnel of the Unit for Laboratory Animal Medicine at the University of Michigan, adhering to the NIH guide for the care and use of laboratory animals (revised 1985).

Motor task

Cats were trained by operant and classical conditioning to perform successive conditional discriminations in a GO/NO-GO reaching task with the right forelimb. The behavioral testing apparatus was contained within a vented box $(90 \times 82 \times 100 \mathrm{~cm})$ to minimize extraneous auditory and visual disturbances. The animals were monitored continuously with a video camera, force platform transducers, contact detectors and accelerometers. The two accelerometers (Entraum EGA-125F-5D, sensitivity range $\pm 5 \mathrm{~g}$ ) were mounted orthogonally on the recording chamber to monitor rostral-caudal and medial-lateral head acceleration. The platform on which the animals sat had a force transducer and contact sensor for each foot (Fig. 1). A touch pad, which was the target of the reaching movement and also monitored by a contact sensor, was placed at a comfortable distance in front of the animal. No head fixation or limb immobilization was used. We minimized physical restraints to encourage natural posture, movements and computational processing in the central nervous system. The animals were free to self-select a comfortable sitting position on the platform, constrained only by a collar that rotated freely and moved vertically over a range of 3 $\mathrm{cm}$.

GO and NO-GO visual cues on an LED display on the target pad were signified by three horizontal lines (GO) or three vertical lines (NO-GO). A computer controlled the motor task. Each trial began with a 2-s control period prior to cue presentation during which the computer monitored animals to ensure that they sat quietly and maintained force platform contact with all four limbs. The control period was designed to ensure a consistent behavioral state prior to cue presentation for the purpose of collecting baseline neuronal and muscle activity during quiet sitting. Failure to remain quietly seated during the control period would result in trial failure. The animals were not aware when the control period began as it was contiguous with the intertrial period and there was no signal to mark its onset. Only after a cue was presented were they aware a trial had started. Within $5 \mathrm{~s}$ after a GO cue, the animals had to touch the target and maintain contact with it for $500-1250 \mathrm{~ms}$. These time constraints were not changed throughout the course of the study and, within the constraints indicated, we did not attempt to train the animals to react or move faster in response to the cues than the natural pattern that they acquired during their course of training. After a NO-GO cue, the animals had to maintain contact with all four touch pads and to refrain from touching the reward delivery cup for 1000-2000 ms. Correct performance in both GO and NO-GO trials was reinforced by a food reward consisting of a bolus of liquefied cat food. To increase motivation, food was partially restricted in the home cages of the animals. Usually, we presented trials until the animals were sated. On occasions when they may not have received enough food during a recording session, they were given supplemental food in their cages. GO or NO-GO trials were intermixed randomly in blocks to obtain ten successful trials of each type whenever possible. A short break of 5-10 min occurred between blocks. One hundred to 300 trials were presented each day in a median of four blocks (range 1-17). 
Eye movement (EOG) or muscle activity (EMG) was recorded in $17 \%$ of testing sessions. EMG electrodes consisting of two stainless steel sterile wires were implanted percutaneously in each muscle prior to a recording session. Muscle activity was amplified, filtered $(30-1000 \mathrm{~Hz})$, integrated and recorded by a computer. EOG electrodes were attached to shaved skin beside each eye prior to the recording session. The signals were recorded differentially and filtered $(0.01-30 \mathrm{~Hz})$ and recorded by the computer.

\section{Lesions}

Two animals received unilateral lesions of the left striatum contralateral to the lifting paw. We used neuronal recording procedures to ensure accurate lesion placement. Striatal stereotaxic boundaries were calibrated in each animal by the boundaries of white and gray matter observed during electrode penetrations. Quinolinic acid $(200 \mathrm{nM})$ in $0.1 \mathrm{M}$ phosphate-buffered saline ( $\mathrm{pH}$ adjusted to 7.4 with $4 \mathrm{~N} \mathrm{NaOH}$ ) was placed in a microsyringe and 30 -gauge cannula. The cannula, in place of an electrode, was guided to the lesion sites on the basis of the corrected stereotaxic information. Multiple (15-17) small injections $(0.25-1.25 \mu \mathrm{l})$ of quinolinic acid produced large unilateral lesions of both the caudate nucleus and putamen while sparing surrounding structures. We injected slowly ( $1 \mu \mathrm{l} / 5 \mathrm{~min})$ and left the cannula in place for an additional $5 \mathrm{~min}$ to prevent back-flow along the electrode track. An injection of $1 \mu \mathrm{l}$ produced a lesion approximately $1 \mathrm{~mm}$ in diameter.

At the end of the study, the extent of the lesion and the location of recording sites were determined from histological reconstructions. The animals were anesthetized deeply with pentobarbital Small electrolytic marking lesions were made and then the animals were perfused intracardially with saline followed by $10 \%$ buffered formalin in $0.9 \%$ saline and $1.5 \%$ potassium ferrocyanide. The brain was removed and cryoprotected with a series of sucrose-formalin solutions (10-30\%). Forty-micrometer serial sections cut on a freezing microtome were floated onto slides and stained with cresyl violet. The cresyl violet stained sections were analyzed by an image processor (Microcomputer Imaging Device, Imaging Research, St. Catherine's, Ont.) to determine the extent of the lesions. Sections taken at regular intervals through the lesion were viewed on the video display terminal and a manually controlled cursor was used to delineate the borders of the nuclei of the lesioned and contralateral striatum. The area within the outlined borders was computed and the ratio of the lesioned to contralateral side was determined.

\section{Analysis of motor behavior}

Motor behavior in contexts apart from the motor task was compared qualitatively before and after the lesion. These evaluations relied on visual and videotaped observations in the behavioral test apparatus during the period between trials (standing and sitting posture, orienting, vocalizations, etc.). In addition, play behavior, social interactions and circumscribed behavioral and neurological tests were evaluated in the home cage or one the open floor in the home cage room. Animals were tested for placing reactions, limb stiffness, attentiveness to laterally placed auditory (jingling keys) or visual stimuli (e.g., food treat), ability to chase and catch food treats thrown in the air or along the floor on their right or left sides, beam walking to follow a food treat and string chasing. Somatosensory attention was assessed by placing matching pieces of masking tape at proximal and distal locations on the right and left limbs and trunk simultaneously (Schallert and Whishaw, 1984). Determining which piece the animal removed first and the elapsed time gave some indication of sensory attentiveness and lateral differences.

Fig. 1 Motor task and center of pressure. Left: behavioral apparatus. The platform has one force transducer $(C)$ and one contact sensor $(E)$ for each limb. The touch target for the right limb $(B)$ also has a contact sensor. Liquefied food rewards were presented at $A$. The center of the pressure axis is illustrated $(D)$ with a single center of pressure $(\mathrm{CoP})$ vector (dashed arrow in lower left quadrant). Middle: $\mathrm{CoP}$ points for one GO trial plotted on the mediallateral and rostral-caudal axes. Each point indicates the location of the CoP measured in that $20-\mathrm{ms}$ interval. The trial starts with CoP near the origin and remains there for some time. The arrow labelled cue points to the $\mathrm{CoP}$ value at the time the cue is presented. This value is superimposed on the other points obtained during the control period. After the cue the animal moves so that the CoP moves backward and slightly to the left. The arrow labelled Release points to the $\mathrm{CoP}$ value at the time the right forepaw is released from the contact sensor. The arrow labelled Touch is the value at the time the right paw makes contact with the target. Right: CoP vector arrays as a function of time. Each row of vectors represents one trial aligned horizontally with time $=0$ at onset of the GO cue. Each vector represents the location of the CoP at the instant of time indicated by the anchor point of the vector along the time axis with a scale of $\pm 50 \mathrm{~mm}$ indicated along the $y$ axis. The last trial, marked with an asterisk, is the one illustrated in the central $x-y$ plot. The open triangle on each line indicates the point at which the change in $\mathrm{CoP}$ reached a maximum $(\mathrm{CoP}$ Vmax). The vertical line indicates the time of paw release and the cross indicates the time of target touch. After the cue, the vector change in length and orientation represents the animal movements from attentive waiting to reaching toward the target (Fig. 2). Since there is a transfer of weight to the target after it is touched, $\mathrm{CoP}$ is no longer defined by force platform data alone and these vectors are indicated by gray lines. After target contact $\mathrm{CoP}$ data should be viewed as providing only temporal information about changes in posture
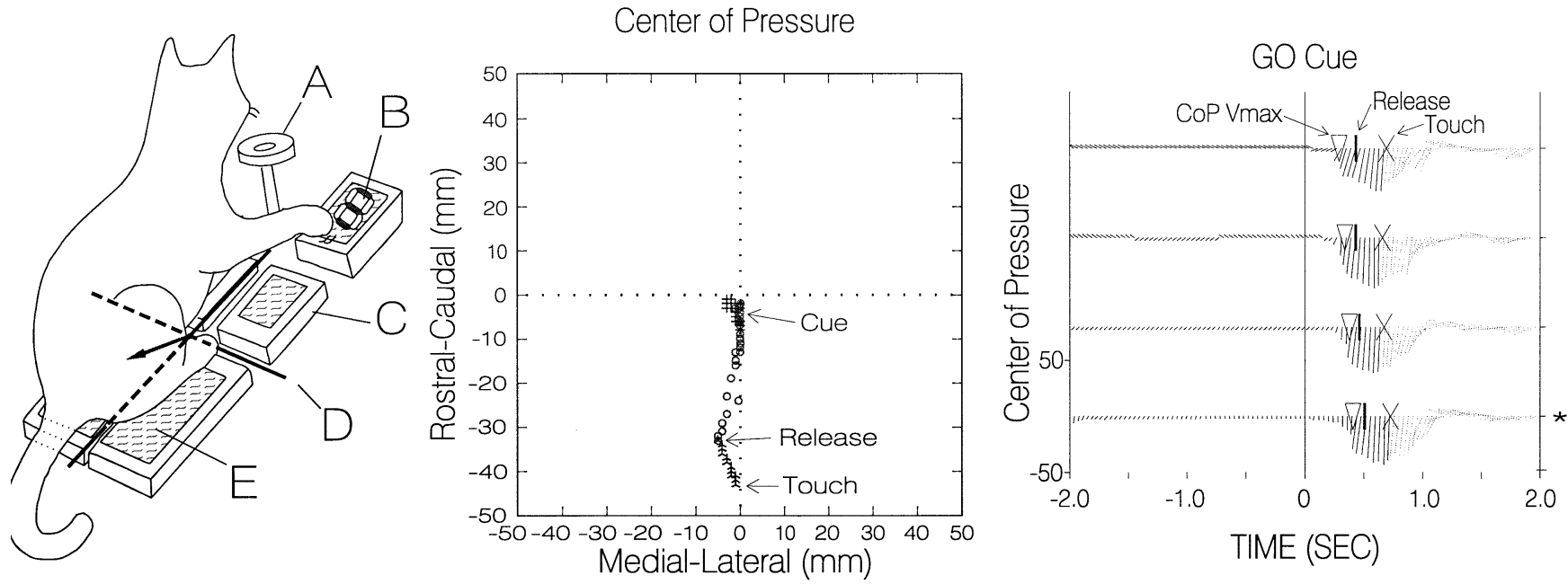
Postural movements, including those that precede paw release in GO trials and movements made in NO-GO trials, were monitored by computing the trajectory of the center of pressure in time. The center of pressure is the $X-Y$ position of the point vertically below the animal's center of mass (Gray 1994). This point moves on the $X-Y$ plane of the force platform as the animal changes posture (Fig. 1, center panel). A computer program calculated and stored the center of pressure at a sampling rate of 10 or $20 \mathrm{~ms}$ for each trial. In GO trials, the center of pressure is defined only until the instant at which the animal touches the target, as weight transfer to the target pad was not considered. We used a separate program to compute the first derivative (velocity) of these data and to determine the onset of movements. The first detectable movement based on a threshold crossing method (a movement change greater than 1 or $2 \mathrm{~mm}$ ) has the disadvantage of being subjected to low level noise and thus prone to large errors. The onset of movement defined by the peak velocity (CoP Vmax) had the distinct advantage of being well defined with a clear time point (Fig. 1, righthand panel) although it is slightly later than the "true" onset of movement. We determined and present data using both methods. The peak detection method picked onset times that were later by a median value of $80 \mathrm{~ms}$.

We evaluated behavioral motor performance in the task by computing mean reaction and movement times and the rates of success and failure in each block of trials. Statistical comparisons of pre- and post-lesion states were made using each animal as its own control. Reaction time to lift the forelimb in cats is determined in part by the biomechanical constraint of quadrupedal stance requiring a shift in the center of mass over a triangle formed by the left front and hind limbs prior to reaching (Gray 1944; Macpherson 1988a, b). In GO trials, reaction time to release (Release RT) was defined as the time between sensory cue presentation and paw release from contact sensor. Movement time (MT) was the time between paw release and target contact. By means of the center of pressure trajectories, we computed analogous reaction times to postural movements in both GO and NO-GO trials The first detectable postural movement after the cue defined the center of pressure onset time (CoP Onset). The time at which the first derivative of center of pressure reached a maximum $(\mathrm{CoP}$ Vmax) defined CoP Vmax reaction time (CoP Vmax RT). In addition to limb movement, we also measured the reaction time from the presentation of the reward cue to the first contact of food delivery cup in both GO and NO-GO trials (Lick RT).

\section{Results}

Cats learned the GO/NO-GO task in approximately 2 months. Up to 5 months of additional training were required to ensure consistently high levels of performance. The prelesion data set consists of 17598 successful and 4541 unsuccessful trials from four animals. An additional 4300 successful and 3460 failed trials were recorded in two animals after a striatal lesion. Pre- and post-lesion comparisons were done with each subject serving as its own control.

\section{Prelesion behavior}

\section{Postural motor behavior}

The general sequence of movements invoked by the task was similar from trial to trial and from animal to animal. The cats watched the stimulus display with intense concentration while waiting for a cue. Occasionally, they swept the tip of their tail back and forth rhythmically in

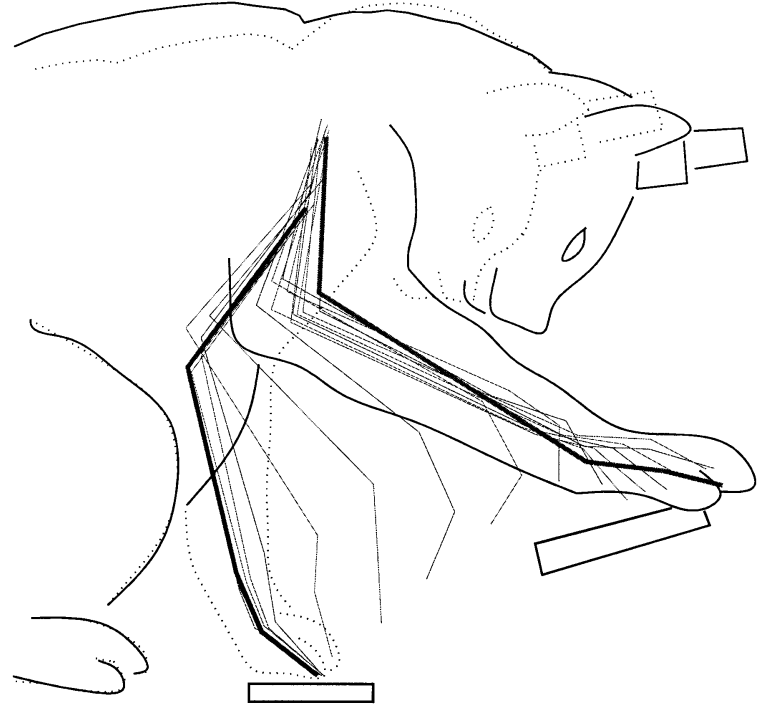

Fig. 2 Forelimb movement in the GO task. A sequence of graphical representations of the forelimb position viewed from the side was made by tracings on plastic transparencies from a video monitor using a frame-by-frame stop motion video recorder. The sequence illustrates the movement from the onset of the GO cue to target contact (reaction time+movement time). Each stick figure shows the location of the limb defined by reference points on the shoulder, elbow, wrist and toes at 33.3-ms intervals (one video frame), with 19 measurements (total time $633 \mathrm{~ms}$ ). Bold stick figures indicate the first (leftmost) and last (right) positions in the movement sequence. A dashed line depicts the outline of the cat at the onset of the cue (first thick figure) and a continuous line shows the cat at the end of the movement. Note the forward movement of the head and trunk, upward movement of the shoulder and the fact that the limb extends above and beyond the target before making final contact. Movement speed is slowest at the beginning and end of the movement as indicated by overlapping figures

the manner of attentive cats stalking prey (Leyhausen 1979); otherwise they sat quietly watching the display. Following a GO cue, a cat would propel its body forward toward the target coincident with a smoothly executed lifting movement of the right forelimb (Fig. 2). The forelimb moved rapidly forward, extending slightly above the endpoint prior to its final placement on the target. This postural change was accompanied by a center of pressure trajectory that moved backward and slightly to the left (Figs. 1, 3). The backward component indicates a downward force by the hindlimbs. The smaller leftward component of the center of pressure trajectory could represent a combination of weight shifting off the right forelimb and/or weight transfer from the right to left hindlimb. This postural change was merged smoothly and skillfully into the subsequent reaching movement. Prior to cue onset, the animals made little or no extraneous movement, but when they did the movements were smaller in amplitude than those following the cue and not correlated temporally with task events (Figs. 1, 3).

In NO-GO trials the only task requirement, which trained animals met easily, was to maintain platform contact with all limbs. Nevertheless, all animals made 


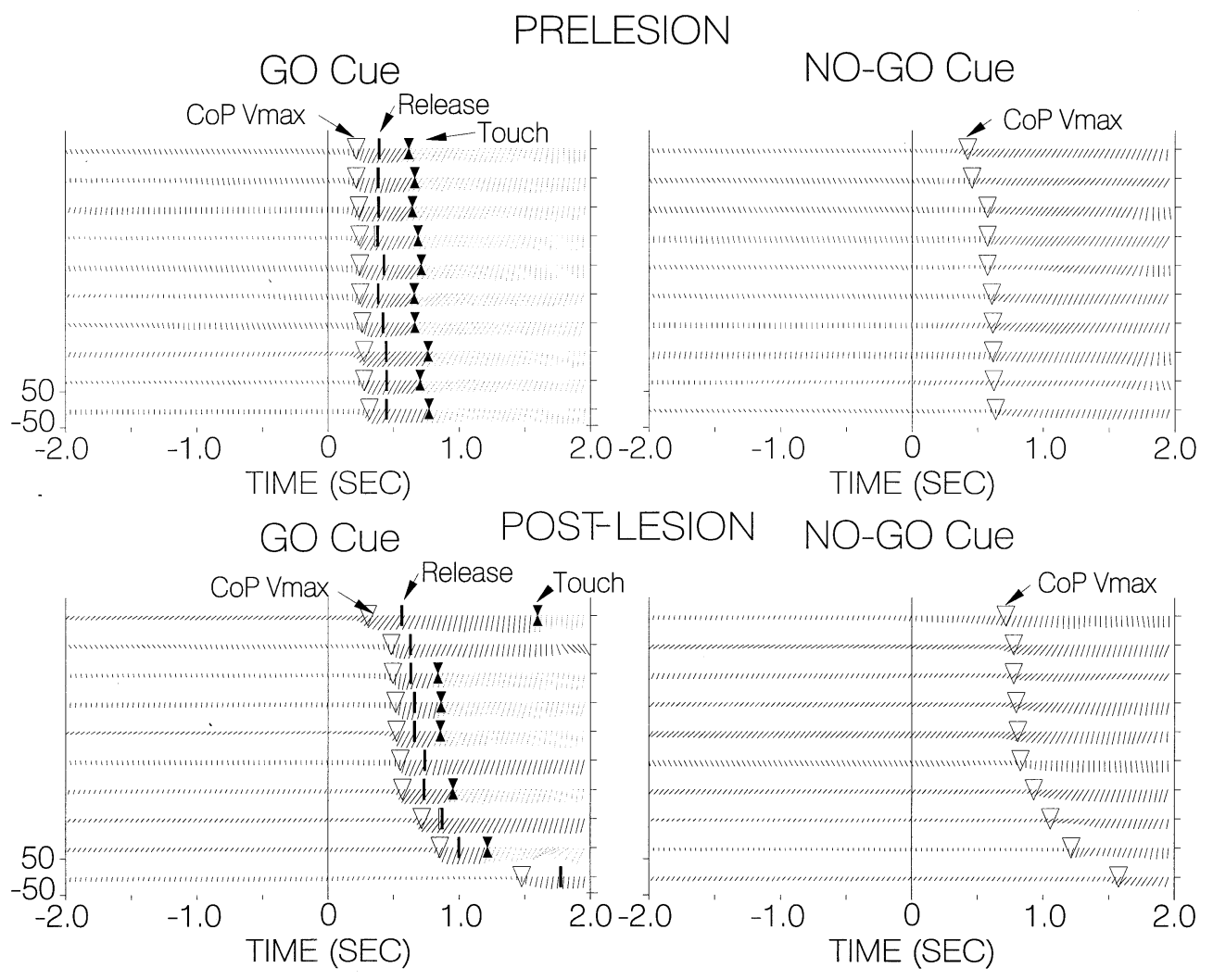

similar postural movements in NO-GO trials as well. The trajectory of the center of pressure resembled that of GO trials (Fig. 3), although the timing differed (see below) and the animals did not lift their forelimb from the right front contact sensor. The final movement in both GO and NO-GO trials was evoked by presentation of the reward cue and food in a metal cup located on the left side of the visual display (Fig. 1).

\section{Head and eye movements}

Head movements associated with the task, like those of limbs and trunk, were coincident with the postural sequence following cue presentations (Fig. 4). Head movements were generally absent or small in amplitude prior to the cue as the animals watched the display steadily. Eye movements were linked closely in time to head movements (Fig. 4). The animals exhibited little or no movement of the eyes prior to the cue, but when they did occur they were concurrent with movements of the head and trunk. Head and eye movements were always detectable after the presentation of a cue.

\section{Muscle activity}

The timing pattern of muscle activity (EMG) corresponded to the postural movements (Fig. 5). EMG recordings were obtained from 26 muscle groups in the trunk, neck and limbs during 22 prelesion recording sessions and 17 muscle groups in 10 post-lesion sessions.
Fig. $3 \mathrm{CoP}$ as function of time for GO (left-hand column) and NO-GO trials (right-hand column) for the same animal (MAT) prior to (top row) and after (bottom row) the lesion. The CoP vectors have the same format as in Fig. 1. The trials are aligned to the instant at which the cue was presented (time $=0$ ). The animals usually sat quietly while waiting for a trial to begin, although in some trials they made small movements as indicated by a change in vector orientation or length (e.g., second trial from top on left). Triggered movements begin shortly after the cue. The onset time of the movement as indicated by CoP Vmax is marked by an open triangle on each trial. The onset of release in GO trials is marked by a vertical line. In the GO trials, an $X$ marks the instant of target contact and subsequent vectors are shown by dashed lines (see Fig. 1). The trials are sorted in the order of increasing reaction time from the cue to the CoP Vmax. Note that the reaction time to the first postural movement is longer in NO-GO than GO trials and that reaction times are longer and more variable after than before the lesion

All the muscles we studied were activated by the task. Patterns of activity were dependent upon the type of trial (GO vs NO-GO). For example, muscles of the right forelimb were activated to lift the limb in GO trials and therefore differed in their activity in NO-GO trials (Fig. 5 ). The relative amplitudes of activity in hindlimb and trunk muscles were correlated with the degree of postural change. The more intensive postural shift and stabilization in GO trials produced larger EMG amplitudes in some muscles. The onset time of activity changes varied slightly among muscle groups and between the two trial types. In trunk and proximal muscles, such as the paraspinal muscles (Fig. 5), activity was detected prior to the initial postural movements ( -50 to $-100 \mathrm{~ms}$ latency). 


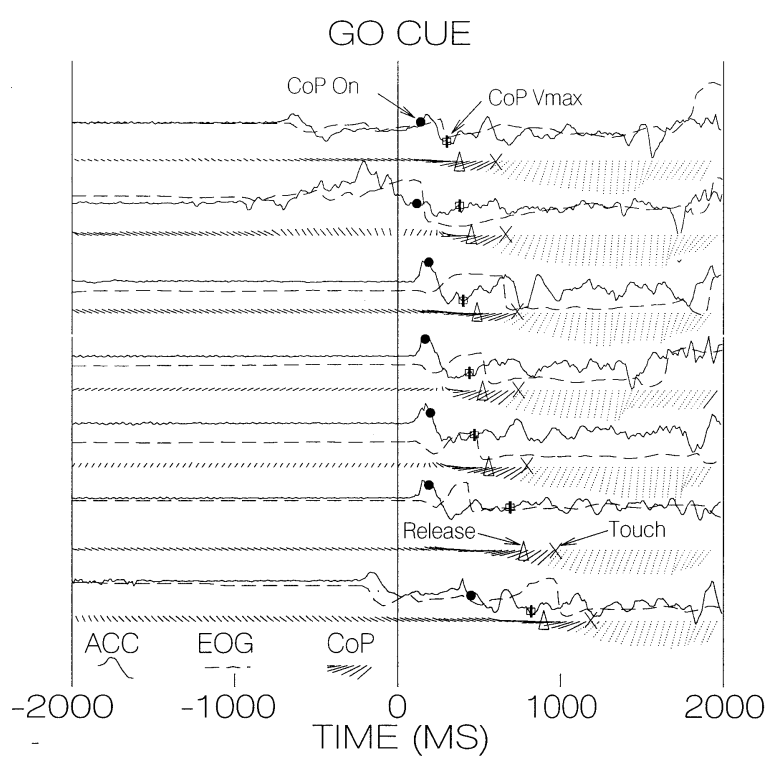

Fig. 4 Eye and head movements. The CoP for GO trials, with the same format as in Fig. 1, along with superimposed traces of eye movements (dashed line) and head acceleration (continuous line) for the same trials. The onset of $\mathrm{CoP}$ movements and the time of CoP Vmax are marked on the acceleration trace by a filled circle and a square with a vertical line, respectively. On the CoP array there is a triangle that marks the onset of release and a cross that marks the contact with the target. In general, the eye and head movements start at the same time or slightly before the time at which postural movements can be detected on the CoP records. This set of trials has examples of head and eye movements that occur during the control period. These also have associated changes in CoP that occur at the same time

\section{Unilateral striatal lesions}

\section{Histological analysis}

The volume of the caudate nucleus and putamen under the chamber on the side of the lesion (left) was reduced and the ventricle was enlarged (Fig. 6). Nearly all the striatum was lesioned. Only the tail of the caudate nucleus was spared. The remaining portions of the head of the caudate were visualized as gliosis. We quantified lesion size by comparing the area of the striatum on the lesion side (animal's left) with that on the control side (right) on cresyl violet stained sections. On the lesioned side, striatal area was approximately half that on the control side within the region comprising the bulk of the striatal volume (AP 12-22, Fig. 7). We extended measurements to more caudal tail regions of the caudate nucleus $(<\mathrm{AP}$ 12 ) in one animal and found less reduction in these regions away from injection sites (Fig. 7). These area measurements estimate neural tissue loss conservatively, as microscopic examination indicated that remaining tissue probably consisted mainly of glial elements.

\section{Post-lesion free-range behavior}

The immediate behavioral effect of the striatal lesion was striking. Within $2 \mathrm{~h}$ of the injection, the animals be- gan to walk in circles to the right away from the side of the unilateral lesion. From 12 to $24 \mathrm{~h}$ following the lesion, circling became slower, discontinuous and less compulsive. At this time the animals would stop, sit, stand and look straight ahead, but still never look or turn to the left side ipsilateral to the lesion. They even resisted forcible turning to the left. This rightward (contralateral) circling could still be detected in a mild form for up to 3 days (Fig. 8). Both visual and tactile placing reactions were initially absent on the contralateral (right) side after the lesion. The ipsilateral side always had normal placing reactions. The affected limb (right) showed increased resistance to passive manipulation of plastic type for about 1 week after the lesion. Within a few days, the placing reactions were detectable again on the contralateral (right) side, but they were slower and they remained slower. Once the period of circling behavior ended, we were able to test attention to visual and auditory stimuli (e.g., jingling keys). For the remainder of the study period, lesioned animals exhibited inattention on the contralateral (right) side. Tactile sensitivity was also reduced on the contralateral (right) side, as judged by the time needed to remove equal-sized pieces of masking tape placed on the fur on matching sites of the left and right limbs and trunk. Inattention and slower placing reactions on the side contralateral to the lesion (right) were some of the few permanent changes that could be detected easily by clinical examination throughout the observation period of several months (Fig. 8).

Within a few days of the lesion there were few obvious abnormalities in spontaneous behavior. Skillfull play movements (climbing in the cages, chasing a ball of string, catching moving objects, crossing and turning on a balance beam, and walking and running on the open floor) recovered completely within 8 days. Even during days 3-8 the deficits were less severe and often difficult to detect (Fig. 8). In contrast to the apparent recovery in free-range behavior, motor task behavior was greatly impaired (see below).

\section{Motor task: pre- and post-lesion behavior}

\section{Prelesion success rate}

Each animal had a unique pattern of GO/NO-GO success rates (Table 1). The overall success rate (all animals, prelesion) was $81.1 \%$ for GO and $80.2 \%$ for NO-GO. Success rates differed significantly between animals (Table 1) and trial types (GO vs NO-GO). Three of four animals had more successful NO-GO trials; one animal had more successful GO trials.

Disallowed limb movements were the most common cause of failed trials after the cue presentations (11.9\%). These failures occurred when the animal lifted their left forelimb or either hindlimb off the contact sensors. In NO-GO trials, lifting the right forelimb also produced trial failure. In $4.4 \%$ of trials a disallowed touch of the food delivery apparatus prior to the reward cue caused failures. In $7.6 \%$ of GO trials the animals did not attempt the reaching movement; however, this usually occurred 
GO

BRACHIALIS RIGHT

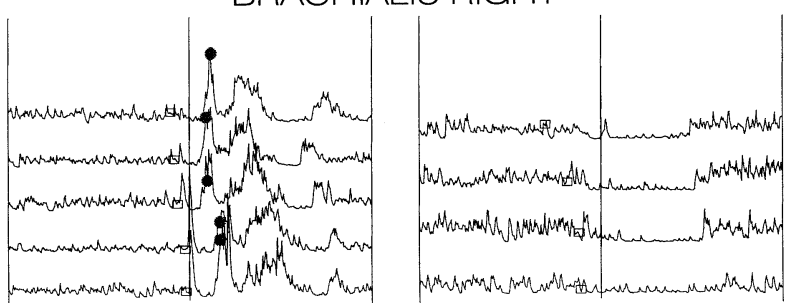

TRICEPS RIGHT
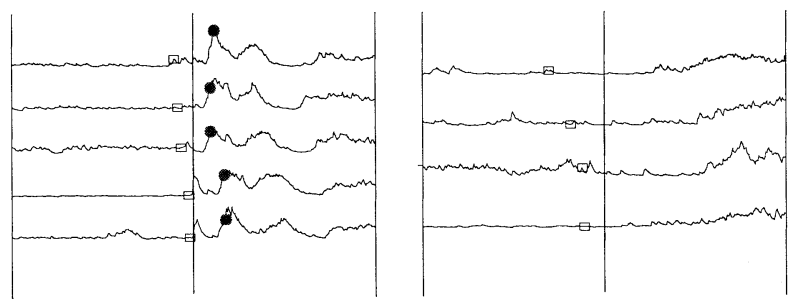

WRIST EXTENSORS RIGHT
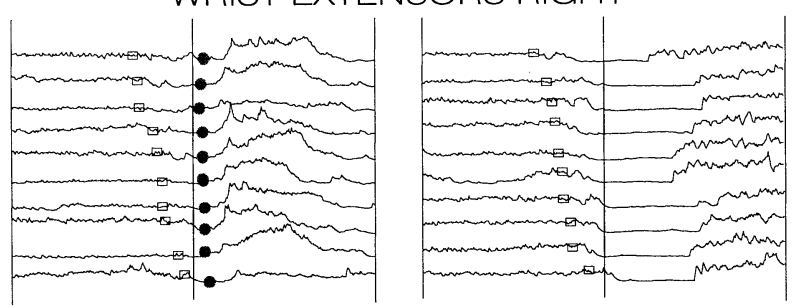

WRIST FLEXORS RIGHT

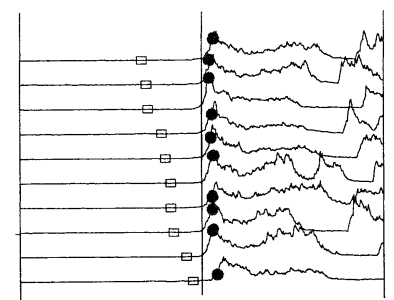

$\mathrm{O}$

TIME (SEC)

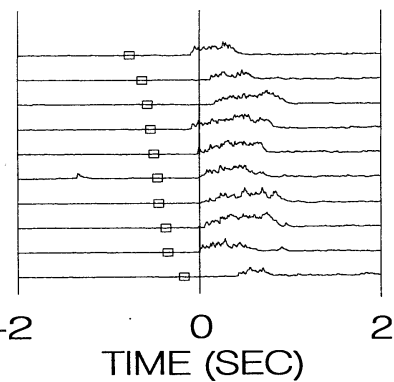

Fig. 5 Muscle activity in GO/NO-GO trials. Recordings from eight different muscle groups are represented in these diagrams. Each set includes trials recorded during GO trials (left) and NOGO trials (right). The trials (rows) are aligned along the time axis $(x$-axis) such that the central line at time $=0$ occurs when the center of pressure change reaches its maximum velocity (see Fig. 1). In each trial, an open square marker to the left of the central time axis indicates the time at which the cue was presented. The trials are sorted in order of decreasing reaction time (top to bottom). The filled circle to the right of the time $=0$ axis in $\mathrm{GO}$ trials indicates when the release from the force platform occurred. In many instances, postural muscles seem to co-contract (e.g., brachialis and triceps), while in other examples there are clear alternations in the activity of agonist-antagonist pairs (e.g., neck and wrist). In some muscles, such as brachialis, the onset of movement leads to cessation of tonic activity. The pattern in brachialis (flexor) prior to the cue may indicate a preparatory stiffening of the limb or posture in which the limb is not fully loaded. In some muscle groups (e.g., paraspinal) the activity can be seen to start rising slightly before the zero line. The latency from the onset of movement as measured by CoP Vmax ranges from -100 to $+100 \mathrm{~ms}$ depending on the muscle group

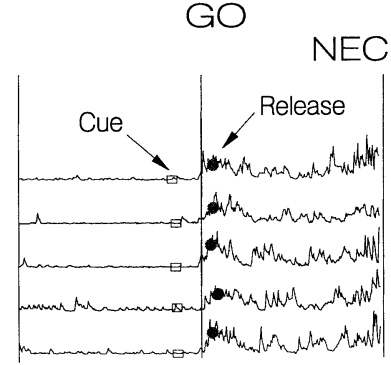

NO-GO

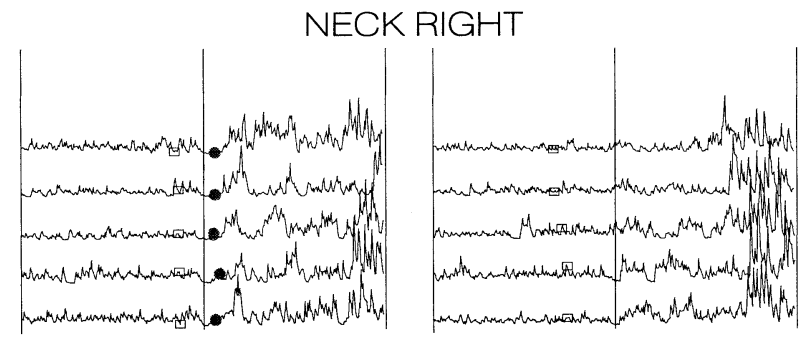

PARASPINAL RIGHT

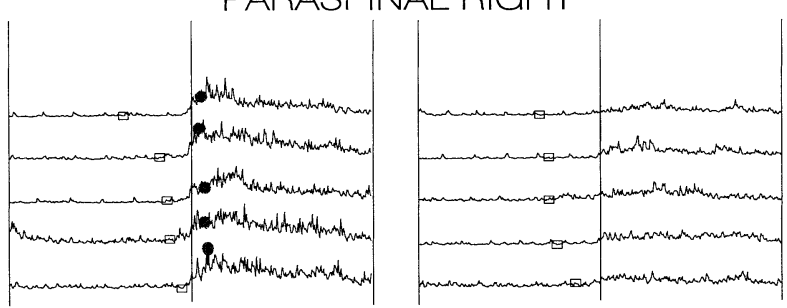

PARASPINAL LEFT

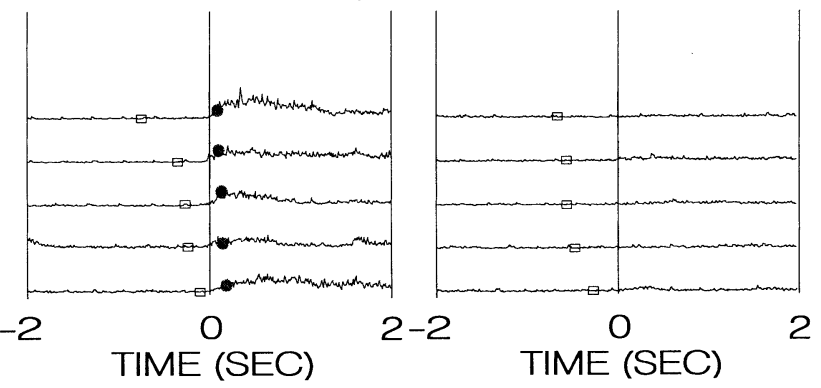

near the end of a recording session. Fully sated animals would sit quietly, ignoring GO cues.

\section{Post-lesion motor task performance}

The lesion produced striking deficits in motor task performance that, in contrast to free-range behavior, persisted throughout the post-lesion study period ( 24 weeks). That the animals remembered the task and the meaning of the cues was clear. They attempted unceasingly to reach in GO trials and to withhold movement in NO-GO trials even in the earliest post-lesion tests when they were greatly impaired. No retraining was ever required. In the first week after the lesion animals could, at best, perform the task with great difficulty. They made preparatory postural movements, but often failed to reach in GO trials (Fig. 9). Although the animals often failed to withhold right limb movement in NO-GO trials, these disallowed movements did not culminate in a reach for the target like those in GO trials. 
Fig. 6 Striatal lesion: two cresyl violet sections through the anterior region of the striatum to illustrate the excitotoxic lesion. The sections represent AP planes 17.5 and 15.0 in one animal. The lesions were on the animal's left side and this is shown on the left side of each section. For this illustration sections were visualized and captured on an image processor. Note the reduced size of the caudate nucleus and putamen on the lesioned side and the enlarged ventricle


17.5

15.0 problem as they would continue to watch the target, waiting for cue presentations. Often, testing could be resumed only when the experimenter touched the extended limb to induce a return to the start position.

\section{Post-lesion success rates}

Overall success rates dropped dramatically after the lesion (Table 1). Because of the increased number of failures, the animals performed more trials (up an average of $49 \%$ ) in each daily recording session after the lesion. Although they failed more trials, both animals (CIC and MAT) maintained their weight close to prelesion values $(\mathrm{CIC}+1.3 \%$ and MAT $-1.2 \%)$. The decrease in success rate was due to more disallowed movements $(30.0 \%$; up $18.1 \%$ ), premature touches of the food delivery apparatus $(7.3 \%$; up $2.9 \%$ ) and failures to reach (10.3\%; up $2.7 \%$ ). Successful performance improved gradually over several weeks, but never to skillful prelesion levels in either GO or NO-GO trials (Fig. 10). One animal recovered faster than the other, but both were still impaired after 24 weeks. The animals had less difficulty with NOGO trials; however, they frequently made the error of lifting their right front paw from the start position. These disallowed movements in NO-GO trials were never followed by a reach to the target; rather, the paw would be momentarily lifted from the force platform and replaced. The fact that the disallowed movements in NO-GO trials were never followed by reaching suggests that they were not simple stimulus discrimination errors.

\section{Prelesion reaction/movement time}

The reaction time from cue to release in GO trials (Table 2) summates all somatic motor activity including postural changes and preparation for reaching. In general, reaction/movement time measures varied widely among animals, although within-subject variability was low. Movement time (Table 2) was shorter than reaction time to re- 
Fig. 8 Behavioral effects of striatal lesions. Behavioral effects from severely impaired black impaired (hatched) to normal (dots) are indicated as a function of time ( $x$-axis) after the lesion. Severe impairment was found only during the first 3 days after the lesion. By 8 days, all behavioral measures except attention to the right side of the body (lesion on left side of animal), placing and task performance had returned to normal. Because of the intense circling in the first 3 days the effects on attentiveness to sensory stimulation were indeterminate

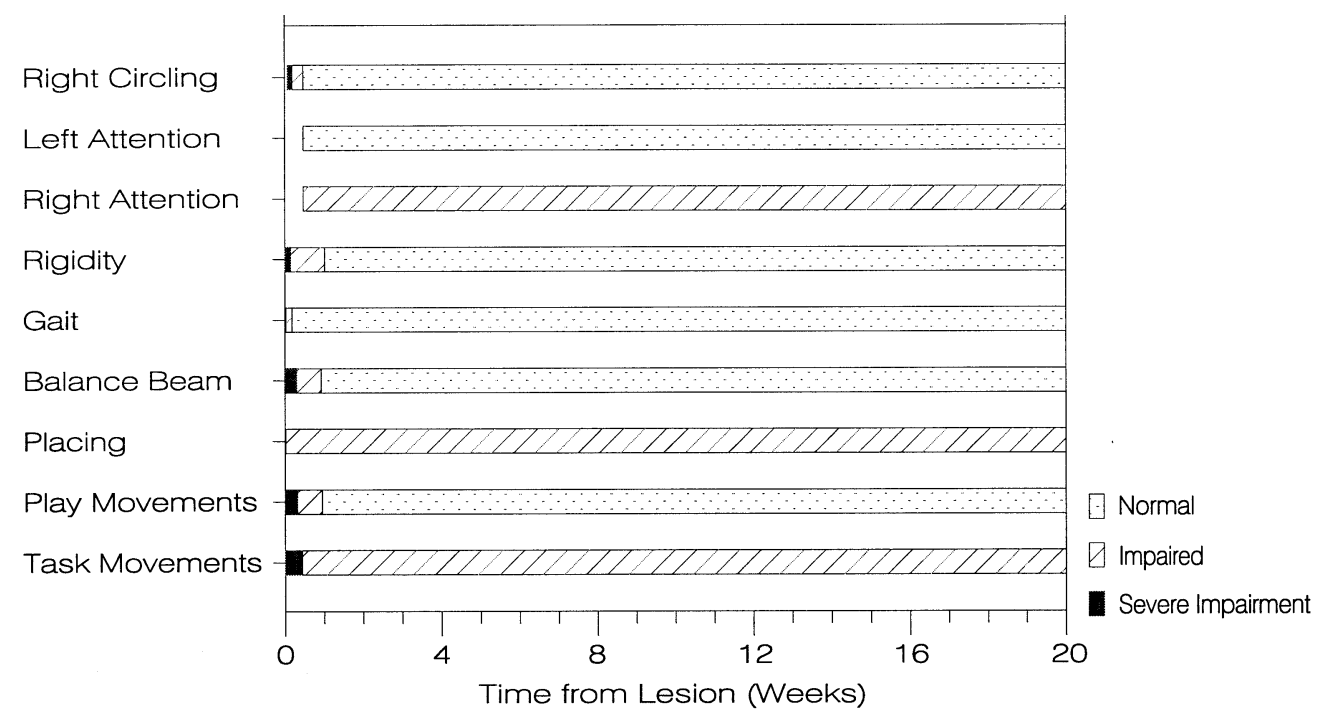

Table 1 Success rates as a percentage of all trials in a GO/NOGO task. Comparisons of success rate between GO and NO-GO trials were significant in all cases pre- and post-lesion (chi-square: ** $P<0.0001$, * $P<0.003)$. Comparisons of pre- and post-lesion states separately for GO and NO-GO trials with each animal serving as its own control showed decreases in both trial types (chisquare: $\dagger P<0.0001)$

\begin{tabular}{llllll}
\hline Animal & \multicolumn{2}{l}{ Success rate $(\%)$} & & \\
\cline { 2 - 3 } & GO & & NO-GO & \\
\cline { 2 - 3 } \cline { 5 - 6 } & Prelesion & Post-lesion & & Prelesion & Postlesion \\
\hline THO & 77.82 & - & & $83.00^{* *}$ & - \\
MIL & 74.58 & - & & $65.92^{* *}$ & - \\
CIC & 80.44 & $43.43^{* * \dagger}$ & & $94.74^{* *}$ & $38.06^{* \dagger}$ \\
MAT & 91.52 & $61.65^{* * \dagger}$ & & $95.52^{* *}$ & $68.53^{* * \dagger}$ \\
\hline
\end{tabular}

lease and varied less. Both reaction time and movement time had a consistent pattern over time, although the latter was more stable (Fig. 11). The reaction time to lick the food delivery cup after the reward cue (Table 2) had a similar range and appearance as the reaction time to reach (Fig. 11).

Analogous indices of reaction time to the onsets of postural adjustments were derived from center of pressure data and in GO and NO-GO trials (Tables 2, 3). Reaction times to the first detectable postural movement in normal animals were as low as $100 \mathrm{~ms}$. Reaction times in NO-GO trials were unpredictable from those in GO trials and significantly different in all cases ( $t$-test: $P<0.0001$ all comparisons). In GO/NO-GO trial comparisons, reaction times to lick the food cup after the reward cue were also significantly different in all animals ( $t$-test: $P<0.0001)$ except one (MAT; Tables 2, 3).

\section{Post-lesion reaction/movement time}

The lesion produced a significant overall slowing of movement in GO trials evidenced by both longer reac- tion time to release and movement time (Table 2). These changes lasted for weeks in both animals (Fig. 11). Reaction times to postural changes in GO and NO-GO trials were also slower after the lesion as measured by the more reliable peak detection method (Tables 2, 3). In contrast to the changes in reaction times to release the limb, changes in reaction times to lick the food cup were less marked after the lesion and in some cases actually became faster on average (MAT: Table 2, Fig. 11; CIC: Table 3).

\section{Previous trial effects: prelesion}

We discovered that the outcome of GO trials exhibited a significant dependence on the immediately preceding trials. The average success rate was higher when following successful $(84.2 \%)$ than failed $(66.6 \%)$ trials $(P<0.01$, chi-square all animals prelesion). Previous trial type also had an effect, with higher average success rates following NO-GO $(85.3 \%)$ than GO $(74.2 \%)$ trials $(P<0.01$, chi-square all animals). The effect of previous trial type outcome was not significant in one of four animals when examined separately (Fig. 12). These previous trial effects were generally not additive over more than one trial; however, after two failed GO trials success rate dropped even further to $31.7 \%$. In contrast to GO trials, the effect of preceding trials on the outcome in NO-GO trials was weak and not significant.

\section{Previous trial effects: post-lesion}

The influence of previous trials on task performance was still detectable and had similar patterns after the lesion (Fig. 12). GO trials were still more successful when they followed successful trials compared with failed trials, and more successful following NO-GO trials than GO trials. Each animal had its own pattern of previous trial effects before the lesion and these persisted in spite of 
Fig. 9 Context dependence. The upper row of video frames illustrates the animal's (CIC) inability to perform appropriate movements in a GO trial 8 days after a striatal lesion. The number at the top of each frame is the duration in seconds measured from the first frame of the sequence. In response to the cue, the animal could only produce an unsuccessful sequence of exaggerated limb extensions that lasted over $5 \mathrm{~s}$ before the trial was aborted by the computer. In the same recording session 5 min later, the lower row of frames shows that the animal was able to produce a reaching movement in response to wiggling fingers on the target in less than $1 \mathrm{~s}$
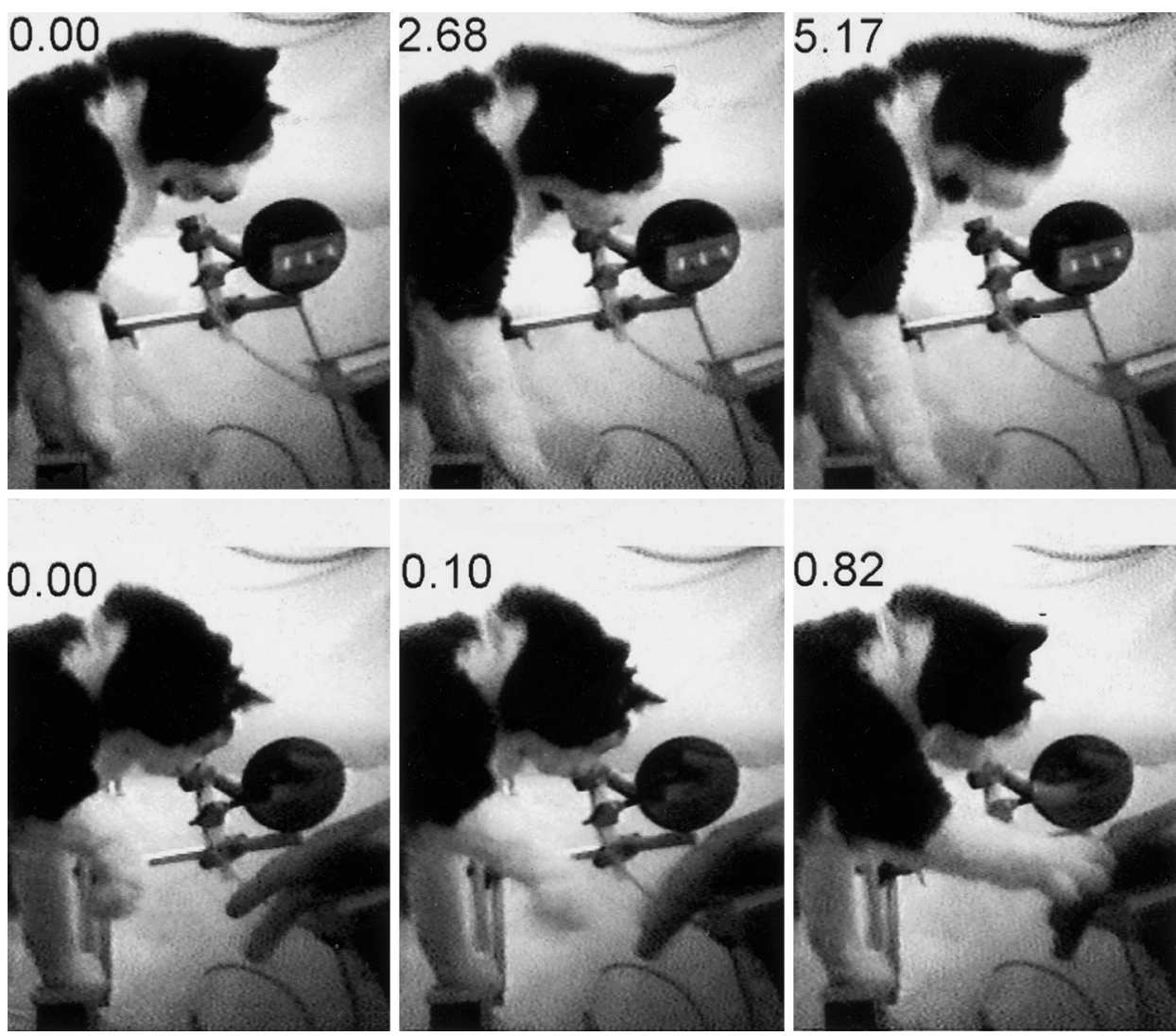

Fig. 10 Task performance. The success rate ( $y$-axis) for each animal (CIC and MAT) is plotted for GO (left) and NO-GO (right) trials. Success rate is indicated as a percentage, where $100 \%$ represents perfect performance and 0 indicates failure in all trials. The rates are plotted as a function of time ( $x$-axis) from the lesion (vertical dashed line at time $=0$, negative values $=$ prelesion). The success rate values are averaged in bins of 2 weeks' duration. There are no data at the time of the lesion or for the first few days as the animals were unable to perform the task. Recovery occurred slowly, although never completely
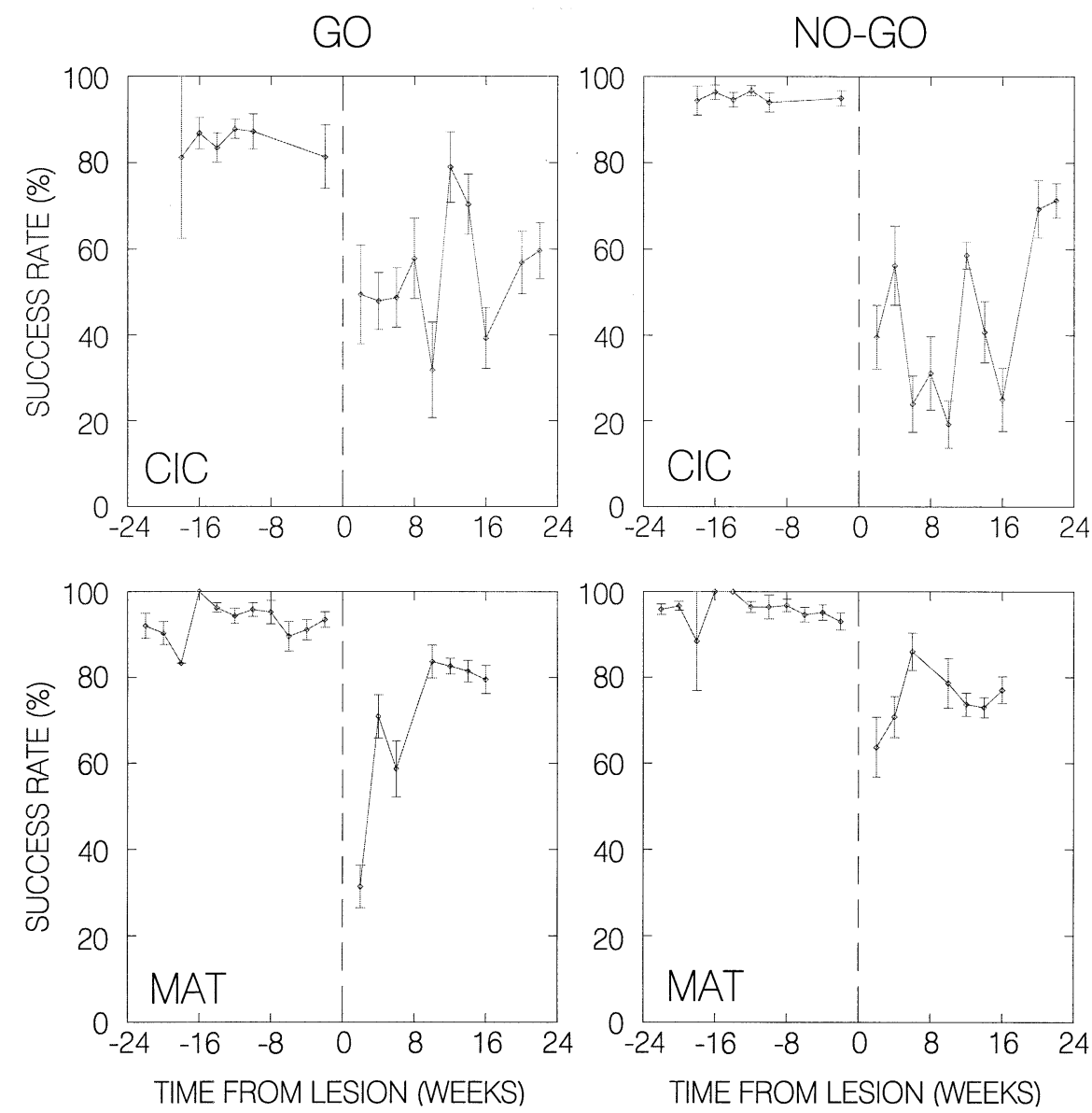
Table 2 GO trial behavioral reaction/movement times. The measurements are means of all successful trials $( \pm$ standard errors of means) in milliseconds. Reaction time measures are from the onset of the GO visual stimulus. CoP Onset Reaction Time is time to the first detectable movement, CoP Vmax Reaction Time is the time at which the first derivative of $\mathrm{CoP}$ pressure reaches a maximum and Release Reaction Time is the time from the stimulus until the paw is released from the starting position. Movement Time is the time between release from the start position until target contact. Lick Reaction Time is the time between the reward cue and first contact to the food apparatus. Two animals (CIC and MAT) had unilateral striatal lesions. Their post-lesion values are shown in the "Post-lesion" column. Two animals (THO and MIL) did not receive lesions. Pre- and post-lesion comparisons were made using each animal as its own control. All comparisons but one were significant $(t$-test: $* P<0.0002, * * P<0.0001$, NS $P>0.05)$

\begin{tabular}{|c|c|c|c|c|c|c|c|c|c|c|}
\hline \multirow[t]{3}{*}{ Animal } & \multicolumn{10}{|c|}{ GO reaction/movement times in milliseconds (mean \pm standard error of mean) } \\
\hline & \multicolumn{2}{|c|}{$\begin{array}{l}\text { CoP Onset Reaction } \\
\text { Time }\end{array}$} & \multicolumn{2}{|c|}{$\begin{array}{l}\text { CoP Vmax Reaction } \\
\text { Time }\end{array}$} & \multicolumn{2}{|c|}{$\begin{array}{l}\text { Release Reaction } \\
\text { Time }\end{array}$} & \multicolumn{2}{|c|}{ Movement Time } & \multicolumn{2}{|c|}{ Lick Reaction Time } \\
\hline & Prelesion & Postlesion & Prelesion & Postlesion & Prelesion & Postlesion & Prelesion & Postlesion & Prelesion & Postlesion \\
\hline THO & $100(1)$ & - & $219(2)$ & - & $427(4)$ & - & $160(1)$ & - & $280(3)$ & - \\
\hline MIL & $301(7)$ & - & $681(11)$ & - & $961(12)$ & - & $368(6)$ & - & 547 (3) & - \\
\hline CIC & 351 (11) & 331 (10) NS & $529(15)$ & $699(17)^{* *}$ & 799 (15) & $994(15)^{* *}$ & $200(2)$ & $793(2) * *$ & $401(7)$ & $476(7)^{* * *}$ \\
\hline MAT & $275(5)$ & $348(9) * *$ & $312(5)$ & $466(10)^{* *}$ & $462(5)$ & $620(5)^{* *}$ & $230(2)$ & $312(11)^{* *}$ & 415 (4) & $392(4) *$ \\
\hline
\end{tabular}
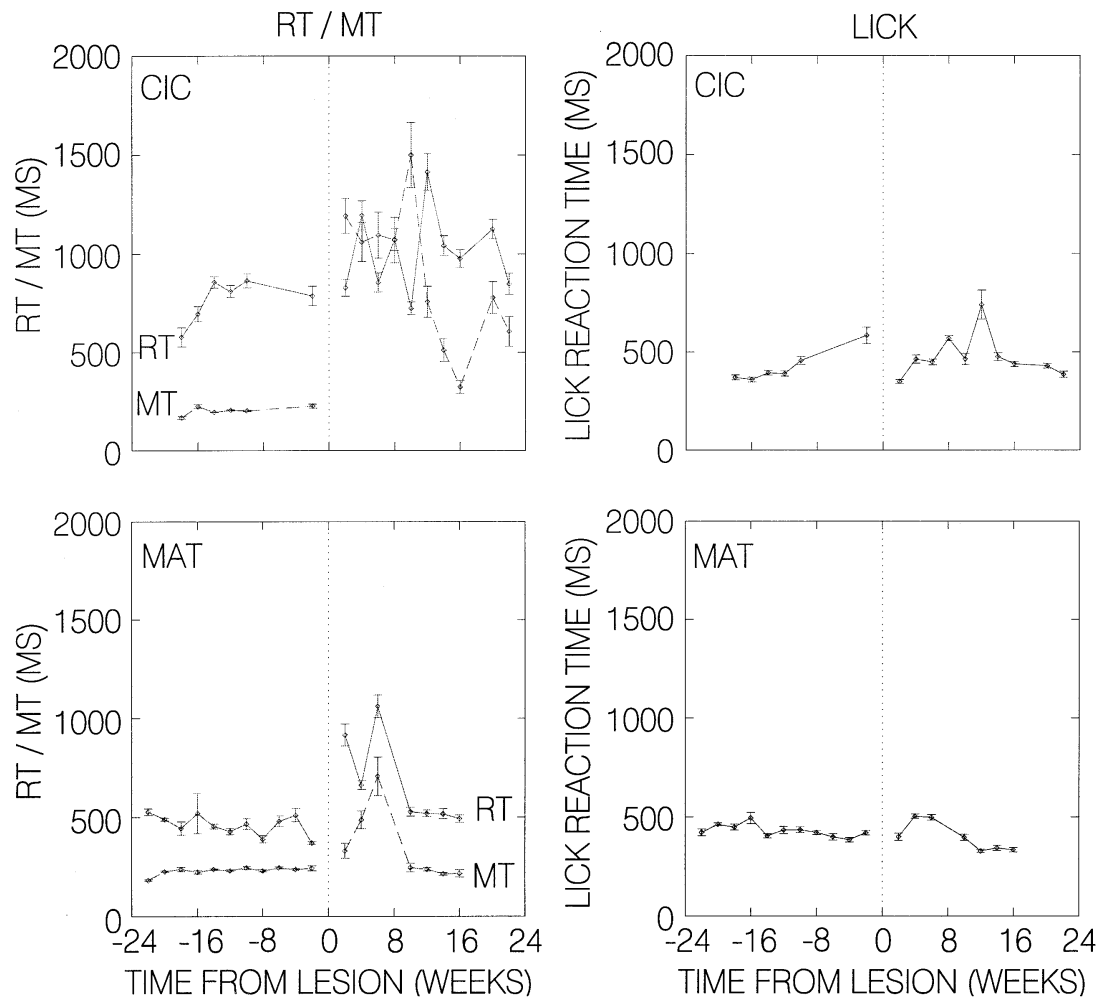

Fig. 11 Reaction/movement time: pre- and post-lesion comparison. Reaction time (RT) to release and movement time (MT) from release to target touch are shown in the left-hand column for two animals (CIC and MAT). The $x$-axis indicates time from 24 weeks before to 24 weeks after the lesion. The $y$-axis indicates the time for either RT (continuous line) or MT (dashed line). RT was greater than MT (top line in the graph). Each point represents the mean RT or MT computed over a 2-week period. Error bars show the standard error of the mean. Note the variability in movement time is very low and error bars are barely visible. After the lesion both RT and MT increase and also exhibit more associated variability. The changes in RT lasted longer and were more severe for one animal (CIC). In contrast to limb movement, the RT to lick the food delivery cup following the presentation of the reward stimulus (right-hand column) was less severely changed by the lesion. The graph format is the same as in the left-hand column

the overall reduction in success. In NO-GO trials, the weak effects observed prior to lesion were magnified and now significant (Fig. 12).

\section{Motor task style}

Each animal had a characteristic "style" or strategy of performance in the motor task exemplified by individual patterns of success and failure (Table 1), reaction and movement times (Table 2) and previous trial effects (Fig. 12). Even though these properties were superimposed on severely degraded performance after the lesion individual patterns were still detectable. For example, one animal (CIC) had faster postural reactions in NO-GO trials 
Table 3 NO-GO behavioral reaction times. The measurements are averages ( \pm standard errors of means) in milliseconds. Reaction time measures are from the onset of the NO-GO visual stimulus to postural movements indices analogous to those in $\mathrm{GO}$ trials. $\mathrm{CoP}$ Onset Reaction Time is time to the first detectable movement and CoP Vmax Reaction Time is the time at which the first derivative of $\mathrm{CoP}$ pressure reaches a maximum. Lick Reaction Time is the time between the reward cue and first contact to the food apparatus. Two animals (CIC and MAT) had unilateral striatal lesions; their post-lesion values are shown. Two animals (THO and MIL) did not receive lesions. Pre- and post-lesion comparisons were made using each animal as its own control. All comparisons were significant $(t$-test: $* P<0.01, * * P<0.0001)$

\begin{tabular}{|c|c|c|c|c|c|c|}
\hline \multirow[t]{2}{*}{ Animal } & \multicolumn{6}{|c|}{ NO-GO postural reaction times in milliseconds (mean \pm standard error of mean) } \\
\hline & \multicolumn{2}{|c|}{ CoP Onset Reaction Time } & \multicolumn{2}{|c|}{ CoP Vmax Reaction Time } & \multicolumn{2}{|c|}{ Lick Reaction Time } \\
\hline THO & $203(4)$ & - & $407(5)$ & - & $419(3)$ & - \\
\hline MIL & $365(7)$ & - & $603(5)$ & - & $478(3)$ & - \\
\hline CIC & $235(7)$ & $194(5)^{* *}$ & $371(8)$ & $405(11)^{*}$ & 481 (10) & $432(7)^{* *}$ \\
\hline MAT & $373(5)$ & $598(5)^{* *}$ & $616(6)$ & $702(6) * *$ & $421(3)$ & $447(4)^{* *}$ \\
\hline
\end{tabular}

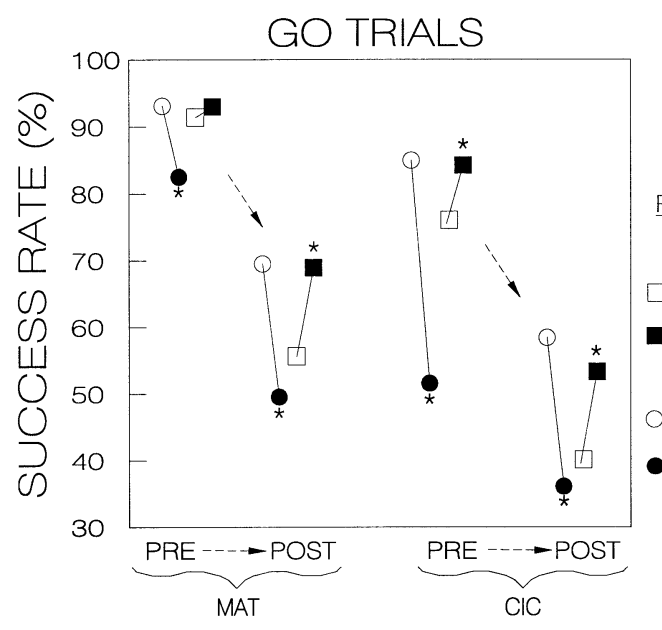

Fig. 12 Previous trial effects. Success rate from $30 \%$ to $100 \%$ is plotted on the $y$-axis. Results obtained from each animal (CIC and MAT) are plotted separately before and after the lesion (Pre and Post) as indicated on the $x$-axis for GO trials (left-hand panel) and NO-GO trials (right-hand panel). Significant differences are marked with an asterisk (chi-square, $P<0.01$ ). Trial types and outcomes are connected by lines for visual grouping. If the preceding trial was a success (open circle), success rate was significantly higher in GO trials than if the preceding trial was a failure (filled circle). If the preceding trial type was a GO trial (open square), success rate in GO trials was lower than if preceded by a NO-GO trial (filled square). NO-GO trials were not significantly influenced by preceding trials prior to the lesion. Note that the GO tria success rates in the two animals were influenced differentially by trial type and outcome before the lesion. These same patterns of preceding trial effects were evident after the lesion although superimposed on lower overall success rates (downward shift indicated by dashed arrow). After the lesion, even NO-GO trials were significantly affected

while the other (MAT) was faster in GO trials (Fig. 13). This relative pattern persisted in spite of slower movement after the lesion.

\section{Discussion}

The consequences of a unilateral striatal lesion on motor behavior were severe. Animals were suddenly, and per-

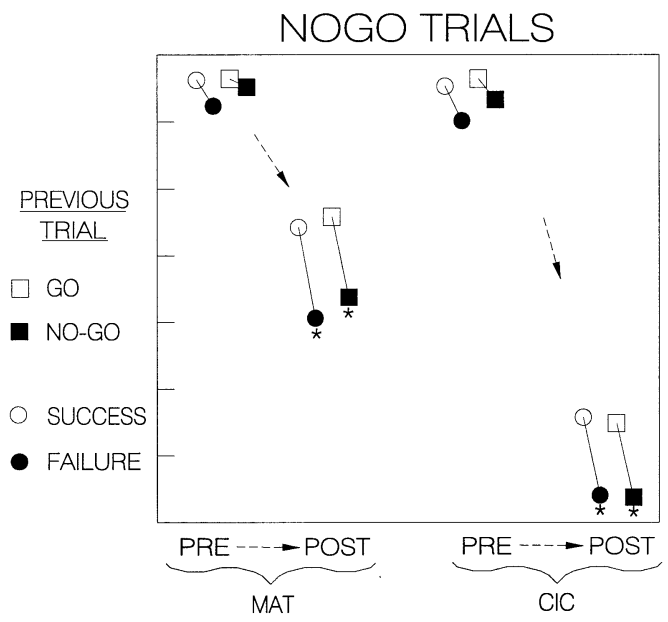

manently, impaired in the performance of a motor task that they once executed swiftly and with great skill. Paradoxically, there was little evidence of motor impairment apart from the learned task even when the movements were similar. The absence of impairment was found even with movements similar to task movements in the behavioral testing apparatus. Outside of the task, only changes in placing reactions and a subtle attention deficit were betrayed by careful clinical examination. The paucity of striatal lesion effects on free-range motor behavior was shown earlier (Villablanca et al. 1976; Benita et al. 1979) and is confirmed by this study. It is possible that other deficits may have eluded our detection in the home cage environment. Free-range and social behavior in cats are highly complex and variable. Also, spontaneous movements may depend upon internal cues that are inaccessible to observers. A more intensive ethological, kinematic and quantitative analysis such as those done in other species (Berridge et al. 1987) will be needed to identify lesion-induced changes in the temporal structure of naturally occurring sequences of motor behavior (Cromwell and Berridge 1996). Our method of calibrating striatal boundaries allowed us to effect a lesion within virtually all of the body of the caudate nucleus and all of the putamen. This means that all striatal circuits except those in the tail of the caudate nucleus were interrupted or dam- 


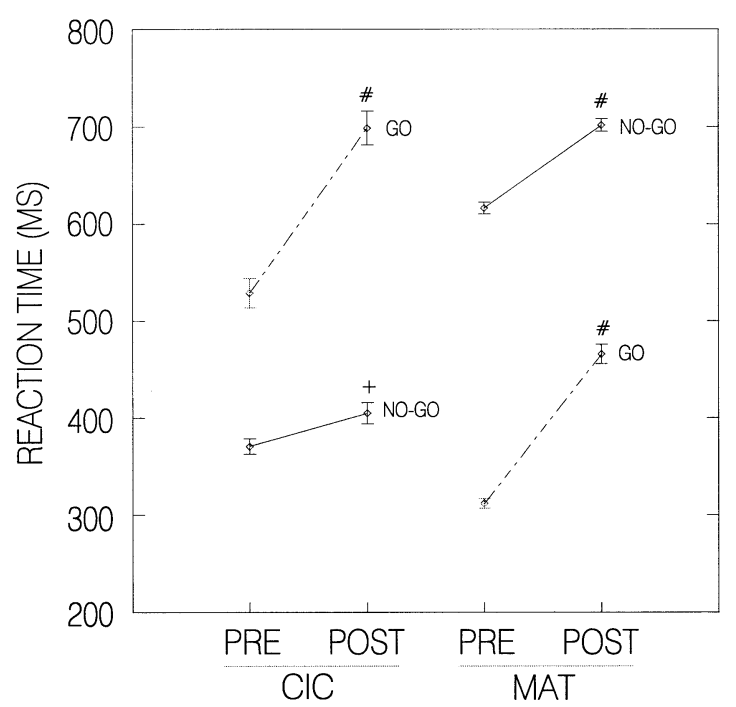

Fig. 13 GO/NO-GO reaction/movement comparison and lesion effects. Mean reaction times and associated standard errors from the cue to the onset of the postural adjustment indexed by CoP Vmax were computed and compared for both GO (dashed lines) and NO-GO (continuous lines) trials. Prelesion values on the left are connected by a line to post-lesion values on the right. Note that one animal (CIC, left) had shorter reaction times in NO-GO trials (continuous line) than in GO trials (dashed line). The second animal (MAT) was faster in GO trials. After the lesion, these relative differences persisted in spite of the significant increases $(t$ test: $+=P<0.01, \#=P<0.0001)$ in reaction time

aged. In particular, the motor circuit would have been severely damaged and could explain the profound effect on motor behavior.

Within the context of the well-learned motor task, the striatal lesion resulted in a marked increase in motor errors due to inappropriate movements. For example, lesioned animals failed to withhold right limb movement in NO-GO trials. In GO trials they failed to withhold extraneous movements of their left forelimb or their hindlimbs. In both trials they were often unable to refrain from licking the food delivery apparatus prior to reward delivery. The disruptive licking of the food apparatus was not evident prior to the lesion. The animals' weights were stable and there was no indication that the animals were hungrier after the lesion. At the beginning of a recording session prior to the lesion animals would have been just as hungry and yet they were able to refrain from premature licking of the food apparatus. Thus, in the absence of striatal control inappropriate movements frequently penetrate to the executive level of the motor system in spite of their adverse behavioral consequences. This finding supports the idea that the basal ganglia have a role in "focusing" information processing in the basal ganglia (Filion et al. 1994) thereby suppressing unwanted movements (Albin et al. 1989).

The intrusion of inappropriate movements was obvious only in the context of the highly practiced motor task. Perhaps the lesion affected the ability of the animals to respond appropriately to sensory cues, although their actions suggested that they remembered the mean- ing of the cues. An essential requirement for fluent execution of a learned sequence of movements is to link cues and movements rapidly and correctly. Thus, an alternative interpretation of the effects of a striatal lesion might be a breakdown in this rapid linkage and could explain why lesioned animals could not suppress inappropriate movements. Further evidence for this process arises from neuronal recording studies in the striatum and pallidum that indicate vigorous activation by the presentation of learned sensory cues (Aldridge et al. 1980a) and activity correlated to movements in a highly learned sequence of movements (Mushiake and Strick 1995).

The quantitative analysis of motor task performance revealed a unique pattern for each animal that was highly consistent from trial to trial and day to day, giving each animal a style recognizable to observers. This individual invariance (Alstermark et al. 1993) conferred singular motor styles that stood in stark relief against a background of intersubject variation. Temporal patterns for motor performances may be determined in part by the manner in which the movement sequence is stored and retrieved from memory (Rosenbaum et al. 1983). The idiosyncratic traits of task performance probably arose during conditioning. It is known that the temporal patterns for movement sequences in humans are robust and persist over long periods of time (Summers 1975). A new finding of the present study is that large unilateral striatal lesions did not abolish the relative motor characteristics unique to individuals, although these features were superimposed on lower success rates and slower movements. This finding supports the idea that motor strategies for the temporal structure of the movement sequences are stored in a distributed fashion in the motor system possibly including the striatum, but probably dependent on the cerebral cortex. Furthermore, temporal encoding is probably not storing absolute timing but instead relative relationships between different motor components.

The striatal lesion produced an overally slowing of movement only during the motor task. Both the preparation and execution of movement were slower as evidenced by longer reaction and movement times. We observed no apparent slowing on skillful movements in free-range behavior, although these movements were less precisely quantified. Still, the slowing of well-learned movements in the motor task was plainly obvious to casual observation.

Reaction times to changes in posture were significantly different in GO and NO-GO trials. This suggests that even the earliest postural movements were dependent on processing visual cue information. We found that head and eye movements were coupled to the movements of the trunk as others have shown (Guitton et al. 1990) and thus could not explain timing differences between GO and NO-GO trials. Thus, the immediate postural changes after a cue were not just alerting responses; rather, these movements were part of overall movement synergies coordinating postural changes of the whole body (Macpherson 1990). Three of the four animals seemed to adopt 
a strategy of preparing in advance for GO trials and restarting preparation if a NO-GO cue appeared, as their postural reaction times were faster in $\mathrm{GO}$ trials. The fact that this relative timing of postural adjustments in $\mathrm{GO}$ and NO-GO trials persists after the striatal lesion further supports the idea of distributed motor memory extending beyond the striatum.

A general reinforcing effect of previous trials was observed and, interestingly, it persisted after the lesion. Following successful trials, success rates were higher and reaction times were faster in comparison with those following unsuccessful trials. A positive motivating influence of successful outcomes on instrumental behavior has been described before (Killeen 1982). We also found that the type of trial, specifically NO-GO trials, could also produce a lingering positive influence on subsequent trials. The reinforcing effect of NO-GO trials may be due to the fact that they were easier to perform than GO trials. The fact that the striatal lesion did not abolish the reinforcing effect of previous trials does not rule out the convergence of motivational and motor mechanisms within the basal ganglia (Schultz et al. 1993); however, it does suggest that evaluation and assignment of positive or negative reinforcing value likely occurs in structures other than the striatum.

The deleterious effects of the striatal lesion were permanent. Even several months after the lesion the animals still performed slower than before the lesion. One animal exhibited more recovery, which may have been due to variation in the lesion in different striatal regions and the corresponding functional control differences between regions (Oberg and Divac 1979). Compensatory changes may occur within other intact structures of the basal ganglia after the lesion. Indeed, there is evidence that after a striatal lesion neuronal activity related to the visual cues and movements in the globus pallidus and subthalamic nucleus exists in the same proportions as before the lesion (Aldridge et al. 1994). In addition, the striatum in the opposite hemisphere does show activity correlated to the performance of sensory cued movement (Jaeger et al. 1993). Future experiments should test well-learned movements on both limbs and bilateral lesions.

What to the basal ganglia contribute to the performance of a learned motor task? Our findings suggest that the striatum facilitates the automatic execution of learned movement strategies, which may be cortically mediated through parallel anatomical loops with the basal ganglia (Alexander et al. 1986; Hoover and Strick 1993). Striatal lesions disrupt the fluent and rapid execution of learned stimulus-response sensorimotor associations, but not their content or details. Neither perceptual processing nor the ability to make voluntary movements depends on the integrity of the striatum. This idea is in keeping with Marsden's hypothesis that the basal ganglia are important in the automatic execution of learned movements (Marsden 1982). Impaired procedural skills in Huntington's and Parkinson's diseases (Saint-Cyr et al. 1988; Knopman and Nissen 1991) further supports this idea. The long-term effects of the lesion may be explained by the loss of function and, possibly, compensatory changes in intact brain and/or release of hierarchically subservient functions in regions once connected to the lesioned structure. This recovery is never complete, underscoring the importance of the striatum for controlling the rapid execution of highly learned sensory cued movements.

Acknowledgements The authors would like to acknowledge the technical assistance of Don Jones of the University of Michigan Space Physics Research Shop and the computing assistance of Eric Walters and Scott Connell. Drs. K.C. Berridge and R.C. Meyer provided helpful comments on the manuscript. Huei-Ming Chai assisted in the preparation of Fig. 2. The research was supported by NIH grants NS31650 (J.W.A.) and NS19613 (S.G.).

\section{References}

Albin RL, Young AB, Penney JB (1989) The functional anatomy of basal ganglia disorders. Trends Neurosci 12:366-375

Aldrigde JW, Anderson RJ, Murphy JT (1980a) The role of the basal ganglia in controlling a movement initiated by a visually presented cue. Brain Res 192:3-16

Aldridge JW, Anderson RJ, Murphy JT (1980b) Sensory motor processing in the caudate nucleus and globus pallidus: a single unit study in behaving primates. Can J Physiol Pharmacol 58: 1192-1201

Aldridge JW, Gilman S, Jones D (1988) A microdrive adapter for chronic single unit recording. Physiol Behav 44:821-823

Aldridge JW, Thompson JF, Gilman S (1994) Neuronal activity related to sensory cues and movement in the subthalamic nucleus of the cat. Soc Neurosci Abstr 20:780

Alexander GE, Crutcher MD (1990) Neural representations of the target (goal) of visually guided arm movements in 3 motor areas of the monkey. J Neurophysiol 64:164-178

Alexander GE, DeLong MR, Strick PL (1986) Parallel organization of functionally segregated circuits linking basal ganglia and cortex. Annu Rev Neurosci 9:357-382

Alstermark B, Lundberg A, Pettersson LG, Tantisira B, Walkowska M (1993) Characteristics of target-reaching in cats. 1: Individual differences and intra-individual constancy. Exp Brain Res 94:279-286

Amalric M, Koob GF (1987) Depletion of dopamine in the caudate nucleus but not in nucleus accumbens impairs reactiontime performance in rats. J Neurosci 7:2129-2134

Anderson ME, Horak FB (1985) Influence of the globus pallidus on arm movements in monkeys. III. Timing of movement-related information. J Neurophysiol 54:433-448

Benecke R, Rothwell JC, Dick JPR, Day BL, Marsden CD (1987) Disturbance of sequential movements in patients with Parkinson's disease. Brain 110:361-380

Benita M, Conde H, Dormont JF, Schmied A (1979) Effects of caudate nucleus cooling on the performance of conditioned movements in cats. Neurosci Lett 14:25-30

Berridge KC, Fentress JC (1987) Disruption of natural grooming chains after striatopallidal lesions. Psychobiology 15:336-342

Berridge KC, Fentress JC, Parr H (1987) Natural syntax rules control action sequence of rats. Behav Brain Res 23:59-68

Brotchie P, Iansek R, Horne MK (1991) Motor function of the monkey globus pallidus. 1. Neuronal discharge and parameters of movement. Brain 114:1667-1683

Brown VJ, Robbins TW (1991) Simple and choice reaction time performance following unilateral striatal dopamine depletion in the rat: impaired motor readiness but preserved response preparation. Brain 114:513-525

Cromwell HC, Berridge KC (1996) Implementation of action sequences by a neostriatal site: a lesion mapping study of grooming syntax. J Neurosci 16:3444-3458

DeLong MR, Georgopoulos AP, Crutcher MD, Mitchell SJ, Richardson RT, Alexander GE (1984) Functional organization of 
the basal ganglia: contributions of single-cell recordings. In: Anonymous functions of the basal ganglia. (Ciba foundation symposium 107) Pitman, London, pp 64-83

Divac I, Markowitsch HJ, Pritzel M (1978) Behavioral and anatomical consequences of small intrastriatal injections of kainic acid in the rat. Brain Res 151:523-532

Filion M, Tremblay L, Matsumura M, Richard H (1994) Dynamic focusing of informational convergence in basal ganglia (in French). Rev Neurol (Paris) 150:627-633

Georgopoulos AP, DeLong MR, Crutcher MD (1983) Relations between parameters of step-tracking movements and single cell discharge in the globus pallidus and subthalamic nucleus of the behaving monkey. J Neurosci 3:1586-1598

Gray J (1994) Studies in the mechanics of the tetrapod skeleton. J Exp Biol 20:88-116

Guitton D, Munoz DP, Galiana HL (1990) Gaze control in the cat: studies and modeling of the coupling between orienting eye and head movements in different behavioral tasks. J Neurophysiol 64:509-531

Hoover JE, Strick PL (1993) Multiple output channels in the basal ganglia. Science 259:819-821

Inase M, Tanji J (1995) Thalamic distribution of projection neurons to the primary motor cortex relative to afferent termina fields from the globus pallidus in the macaque monkey. J Comp Neurol 353:415-426

Jaeger D, Gilman S, Aldridge JW (1993) Primate basal ganglia activity in a precued reaching task: preparation for movement. Exp Brain Res 95:51-64

Jaeger D, Gilman S, Aldridge JW (1995) Neuronal activity in the striatum and pallidum of primates related to the execution of externally cued reaching movements. Brain Res 694:111-127

Killeen PR (1982) Incentive theory. II. Models for choice. J Exp Anal Behav 38:217-232

Knopman D, Nissen MJ (1991) Procedural learning is impaired in Huntington's disease: evidence from the serial reaction time task. Neuropsychologia 29:245-254

Leyhausen P (1979) Cat behavior: the predatory and social behavior of domestic and wild cats. Garland STPM Press, New York

Liles SL, Davis GD (1969) Athetoid and choreiform hyperkinesias produced by caudate lesions in the cat. Science 164:195-197

Macpherson JM (1988a) Strategies that simplify the control of quadrupedal stance. I. Forces at the ground. J Neurophysiol 60:204:217

Macpherson JM (1988b) Strategies that simplify the control of quadrupedal stance. II. Electromyographic activity. J Neurophysiol 60:218-231

Macpherson JM (1990) How flexible are muscle synergies? In: Humphrey DR, Freund HJ (eds) Motor control: concepts and issues. Wiley, New York, pp 33-47
Marsden CD (1982) The mysterious motor function of the basal ganglia: the Robert Wartenberg lecture. Neurology 32:514-539

Mink JW, Thach WT (1991) Basal ganglia motor control. 1. Nonexclusive relation of pallidal discharge to five movement modes. J Neurophysiol 65:273-300

Mushiake H, Strick PL (1995) Pallidal neuron activity during sequential arm movements. J Neurophysiol 74:2754-2758

Nauta HJW (1986) In relationship of the basal ganglia to the limbic system. In: Vinken PJ, Bruyn GW, Klawans HL (eds) Handbook of clinical neurology. Extrapyramidal disorders. Elsevier, Amsterdam, pp 19-31

Oberg RGE, Divac I (1979) "Cognitive" functions of the neostriatum. In: Divac I, Oberg RGE (eds) The neostriatum. Pergamon Press, Oxford, pp 291-313

Parent A, Hazrati LN (1995) Functional anatomoy of the basal ganglia. I. The cortico-basal ganglia-thalamo-cortical loop. Brain Res Brain Res Rev 20:91-127

Roland PE (1980) Supplementary motor area and other cortical areas in organization of voluntary movements in man. J Neurophysiol 43:118-136

Rosenbaum DA, Kenny SB, Derr MA (1983) Hierarchical control of rapid movement sequences. J Exp Psychol Hum Percept Perform 9:86-102

Sachdev RNS, Gilman S, Aldridge JW (1991) Bursting properties of units in cat globus pallidus and entopeduncular nucleus: the effect of excitotoxic striatal lesions. Brain Res 549:194-204

Saint-Cyr JA, Taylor AE, Lang AE (1988) Procedural learning and neostriatal dysfunction in man. Brain 111:941-959

Schallert T, Whishaw IQ (1984) Bilateral cutaneous stimulation of the somatosensory system in hemidecorticate rats. Behav Neurosci 98:518-540

Schell GR, Strick PL (1984) The origin of thalamic inputs to the arcuate premotor and supplementary motor areas. J Neurosci 4:539-560

Schultz W, Apicella P, Ljungberg T (1993) Responses of monkey dopamine neurons to reward and conditioned stimuli during successive steps of learning a delayed response task. J Neurosci 13:900-913

Stelmach GE, Worringham CJ, Strand EA (1986) Movement preparation in Parkinson's disease: the use of advance information. Brain 109:1179-1194

Summers JJ (1975) The role of timing in motor representation. J Mot Behav 7:229-241

Villablanca JR, Marcus RJ, Olmstead CE (1976) Effects of caudate nuclei or frontal cortical ablations in cats. I. Neurology and gross behavior. Exp Neurol 52:389-420 